\title{
Conservation of the noetherianity by perfect transcendental field extensions
}

Magdalena Fernández Lebrón and Luis Narváez Macarro

\begin{abstract}
Let $k$ be a perfect field of characteristic $p>0, k(t)_{\text {per }}$ the perfect closure of $k(t)$ and $A$ a $k$-algebra. We characterize whether the ring

$$
A \otimes_{k} k(t)_{p e r}=\bigcup_{m \geq 0}\left(A \otimes_{k} k\left(t^{\frac{1}{p^{m}}}\right)\right)
$$

is noetherian or not. As a consequence, we prove that the ring $A \otimes_{k}$ $k(t)_{p e r}$ is noetherian when $A$ is the ring of formal power series in $n$ indeterminates over $k$.
\end{abstract}

\section{Introduction}

Motivated by the generalization of the results in [7] (for the case of a perfect base field $k$ of characteristic $p>0$ ) in this paper we study the conservation of noetherianity by the base field extension $k \rightarrow k(t)_{p e r}$, where $k(t)_{p e r}$ is the perfect closure of $k(t)$. Since this extension is not finitely generated, the conservation of noetherianity is not clear a priori for $k$-algebras which are not finitely generated.

Our main result states that $k(t)_{\text {per }} \otimes_{k} A$ is noetherian if and only if $A$ is noetherian and for every prime ideal $\mathfrak{p} \subset A$ the field $\bigcap_{m>0} Q t(A / \mathfrak{p})^{p^{m}}$ is algebraic over $k$ (see theorem 3.6). In particular, we are able to apply this result to the case where $A$ is the ring of formal power series in $n$ indeterminates over $k$.

We are indebted to J. M. Giral for giving us the proof of proposition 2.5 and for other helpful comments.

2000 Mathematics Subject Classification: 13E05, 13B35, 13A35.

Keywords: Perfect field, power series ring, noetherian ring, perfect closure, complete local ring. 


\section{Preliminaries and notations}

All rings and algebras considered in this paper are assumed to be commutative with unit element. If $B$ is a ring, we shall denote by $\operatorname{dim}(B)$ its Krull dimension and by $\Omega(B)$ the set of its maximal ideals. We shall use the letters $K, L, k$ to denote fields and $\mathbb{F}_{p}$ to denote the finite field of $p$ elements, for $p$ a prime number. If $\mathfrak{p} \in \operatorname{Spec}(B)$, we shall denote by ht(p) the height of $\mathfrak{p}$. Remember that a ring $B$ is said to be equicodimensional if all its maximal ideals have the same height. Also, $B$ is said to be biequicodimensional if all its saturated chains of prime ideals have the same length.

If $B$ is an integral domain, we shall denote by $Q t(B)$ its quotient field.

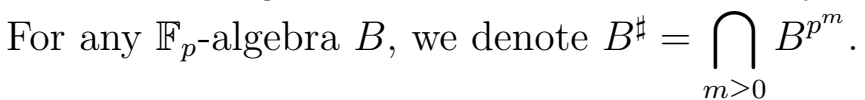

We shall first study the contraction-extension process for prime ideals relative to the ring extension $K[t] \subset K\left[t^{\frac{1}{p}}\right], K$ being a field of characteristic $p>0$.

Let us recall the following well known result (cf. for example [4, th. 10.8]):

Proposition 1.1 Let $K$ be a field of characteristic $p>0$. Let $g(X)$ be a monic polynomial of $K[X]$. Then, the polynomial $f(X)=g\left(X^{p}\right)$ is irreducible in $K[X]$ if and only if $g(X)$ is irreducible in $K[X]$ and not all its coefficients are in $K^{p}$.

From the above result, we deduce the following corollary.

Corollary 1.2 Let $K$ be a field of characteristic $p>0$. Let $P$ be a non zero prime ideal in $K\left[t^{\frac{1}{p}}\right]$ and let $F(t) \in K[t]$ be the monic irreducible generator of the contraction $P^{c}=P \cap K[t]$. Then the following conditions hold:

1. If $F(t)=a_{0}^{p}+a_{1}^{p} t+\cdots+t^{d} \in K^{p}[t]$, then $P=\left(a_{0}+a_{1} t^{\frac{1}{p}}+\cdots+t^{\frac{d}{p}}\right)$.

2. The equality $P=P^{c} K\left[t^{\frac{1}{p}}\right]$ holds if and only if $F(t) \notin K^{p}[t]$.

\section{Proof:}

1. Consider the polynomial $G(\tau)=a_{0}+a_{1} \tau+\cdots+\tau^{d} \in K[\tau]\left(\tau=t^{\frac{1}{p}}\right)$ and the ring homomorphism $\mu: K[\tau] \rightarrow K[t]$ defined by

$$
\mu\left(\sum a_{i} \tau^{i}\right)=\sum a_{i}^{p} t^{i}
$$

From the identity $\mu(G)=F$ we deduce that $G(\tau)$ is irreducible. Since $G\left(t^{\frac{1}{p}}\right)^{p}=F(t) \in P$, we deduce that $G\left(t^{\frac{1}{p}}\right) \in P$ and then $P=\left(G\left(t^{\frac{1}{p}}\right)\right)$. 
2. The equality $P=P^{c} K\left[t^{\frac{1}{p}}\right]$ means that $F(t)=F\left(\tau^{p}\right) \in K[\tau]$ generates the ideal $P$, but that is equivalent to saying that $F\left(\tau^{p}\right)$ is irreducible in $K[\tau]$. To conclude, we apply proposition 1.1.

For each $k$-algebra $A$, we define $A(t):=k(t) \otimes_{k} A$. We also consider the field extension

$$
k_{(\infty)}=\bigcup_{m \geq 1} k\left(t^{\frac{1}{p^{m}}}\right)
$$

If $k$ is perfect, $k_{(\infty)}$ coincides with the perfect closure of $k(t), k(t)_{p e r}$.

For the sake of brevity, we will write $t_{m}=t^{\frac{1}{p^{m}}}$. We also define

$$
A_{(m)}:=A\left(t_{m}\right):=A \otimes_{k} k\left(t_{m}\right)=A(t) \otimes_{k(t)} k\left(t_{m}\right), \quad A_{[m]}:=A\left[t_{m}\right]
$$

and

$$
A_{(\infty)}:=A \otimes_{k} k_{(\infty)}=\bigcup_{m \geq 0} A_{(m)}, \quad A_{[\infty]}:=\bigcup_{m \geq 0} A\left[t_{m}\right] .
$$

Each $A_{(m)}$ (resp. $\left.A_{[m]}\right)$ is a free module over $A(t)$ (resp. over $\left.A[t]\right)$ of rank $p^{m}$ (because $\left(t_{m}\right)^{p^{m}}-t=0$ ).

For each prime ideal $P$ of $A_{(\infty)}$ we denote $P_{[\infty]}:=P \cap A_{[\infty]}, P_{[m]}:=$ $P \cap A_{[m]} \in \operatorname{Spec}\left(A_{[m]}\right)$ and $P_{(m)}:=P \cap A_{(m)} \in \operatorname{Spec}\left(A_{(m)}\right)$.

In a similar way, if $Q$ is a prime ideal of $A_{[\infty]}$ we denote $Q_{[m]}:=Q \cap A_{[m]} \in$ $\operatorname{Spec}\left(A_{[m]}\right)$. We have:

- $P=\bigcup_{m \geq 0} P_{(m)}, P_{[\infty]}=\bigcup_{m \geq 0} P_{[m]}, \quad\left(\right.$ resp. $\left.Q=\bigcup_{m \geq 0} Q_{[m]}\right)$.

- $P_{(n)} \cap A_{(m)}=P_{(m)}$ and $P_{[n]} \cap A_{[m]}=P_{[m]}$ for all $n \geq m$ (resp. $Q_{[n]} \cap$ $A_{[m]}=Q_{[m]}$ for all $\left.n \geq m\right)$.

The following properties are straightforward:

1. The $k$-algebras $A_{[m]}$ (respectively $\left.A_{(m)}\right)$ are isomorphic to each other.

2. If $S_{m}=k\left[t_{m}\right]-\{0\}$, then $A_{(m)}=S_{m}^{-1} A_{[m]}$.

3. Since $\left(S_{m}\right)^{p^{m}} \subset S_{0} \subset S_{m}$, we have $A_{(m)}=S_{0}^{-1} A_{[m]}$ for $m \geq 0$. Consequently $A_{(\infty)}=S_{0}^{-1} A_{[\infty]}$.

4. If $A$ is a domain (integrally closed), then $A_{[m]}$ and $A_{(m)}$ are domains (integrally closed) for all $m \geq 0$ or $m=\infty$.

5. If $A$ is a noetherian k-algebra, then $A_{[m]}$ and $A_{(m)}$ are noetherian rings, for every $m \geq 0$. 
6. If $A=k[\underline{X}]=k\left[X_{1}, \ldots, X_{n}\right]$, then $A_{[\infty]}$ is not noetherian (the ideal generated by the $t_{m}, m \geq 0$, is not finitely generated).

7. If $I \subset A$ is an ideal, then $(A / I)_{(\infty)}=A_{(\infty)} / A_{(\infty)} I$.

8. If $T \subset A$ is a multiplicative subset, then $\left(T^{-1} A\right)_{(\infty)}=T^{-1} A_{(\infty)}$.

9. If $A=k[\underline{X}]$, then $A_{(\infty)}=k_{(\infty)}[\underline{X}]$, hence $A_{(\infty)}$ is noetherian. Moreover, $A_{(\infty)}$ is noetherian for every finitely generated $k$-algebra $A$.

The main goal of this paper is to characterize whether the $\operatorname{ring} A_{(\infty)}$ is noetherian (see theorem 3.6 and corollary 3.8).

Proposition 1.3 With the above notations, the following properties hold:

1. The extensions $A_{[m-1]} \subset A_{[m]}$ and $A_{(m-1)} \subset A_{(m)}$ are finite and free, and therefore integral and faithfully flat.

2. The corresponding extensions to their quotient fields are purely inseparable.

Proof: Straightforward.

Corollary $1.4 A_{[\infty]}$ (resp. $\left.A_{(\infty)}\right)$ is integral and faithfully flat over each $A_{[m]}$ (resp. over each $A_{(m)}$ ).

From the properties above, we obtain the following lemmas:

Lemma 1.5 Let $P^{\prime} \subseteq P$ be prime ideals of $A_{(\infty)}$ (resp. of $\left.A_{[\infty]}\right)$. The following conditions are equivalent:

(a) $P^{\prime} \subsetneq P$

(b) There exists an $m \geq 0$ such that $P_{(m)}^{\prime} \subsetneq P_{(m)}$ (resp. $\left.P_{[m]}^{\prime} \subsetneq P_{[m]}\right)$.

(c) For every $m \geq 0, P_{(m)}^{\prime} \subsetneq P_{(m)}$ (resp. $\left.P_{[m]}^{\prime} \subsetneq P_{[m]}\right)$.

Lemma 1.6 Let $P$ prime ideal of $A_{(\infty)}$ (resp. of $\left.A_{[\infty]}\right)$. The following conditions are equivalent:

(a) $P$ is maximal.

(b) $P_{(m)}$ (resp. $P_{[m]}$ ) is maximal for some $m \geq 0$.

(c) $P_{(m)}$ (resp. $\left.P_{[m]}\right)$ is maximal for every $m \geq 0$. 
Corollary 1.7 With the notations above, for every prime ideal $P$ of $A_{(\infty)}$ we have $\operatorname{ht}(P)=\operatorname{ht}\left(P_{(m)}\right)=\operatorname{ht}\left(P_{[m]}\right)$ for all $m \geq 0$. Moreover, $\operatorname{dim}\left(A_{(\infty)}\right)=$ $\operatorname{dim}\left(A_{(m)}\right)$.

Proof: Since flat ring extensions satisfy the "going down" property, corollary 1.4 implies that $\mathrm{ht}\left(P \cap A_{(m)}\right) \leq \mathrm{ht}(P)$. By corollary 1.4 again, $A_{(\infty)}$ is integral over $A_{(m)}$, then $\operatorname{ht}(P) \leq \operatorname{ht}\left(P \cap A_{(m)}\right)$.

The equality $\operatorname{ht}\left(P_{(m)}\right)=\operatorname{ht}\left(P_{[m]}\right)$ comes from the fact that $A_{(m)}$ is a localization of $A_{[m]}$.

The last relation is a standard consequence of the "going up" property.

Remark 1.8 Corollary 1.7 remains true if we replace $A_{(m)} \subset A_{(\infty)}$ by $A_{[m]} \subset A_{[\infty]}$.

Corollary 1.9 With the notations above, for every $Q \in \operatorname{Spec}\left(A_{(m)}\right)$ there is a unique $\widetilde{Q} \in \operatorname{Spec}\left(A_{(m+1)}\right)$ such that $\widetilde{Q}^{c}=Q$. Moreover, the ideal $\widetilde{Q}$ is given by $\widetilde{Q}=\left\{y \in A_{(m+1)} \mid y^{p} \in Q\right\}$.

Proof: This is an easy consequence of the fact that $\left(A_{(m+1)}\right)^{p} \subset A_{(m)}$.

Corollary 1.10 Let us assume that $A$ is noetherian and for every maximal ideal $\mathfrak{m}$ of $A$, the residue field $A / \mathfrak{m}$ is algebraic over $k$. Then for every $m \geq 0$ we have:

1. $\operatorname{dim}\left(A_{[\infty]}\right)=\operatorname{dim}\left(A_{[m]}\right)=\operatorname{dim}(A[t])=n+1$.

2. $\operatorname{dim}\left(A_{(\infty)}\right)=\operatorname{dim}\left(A_{(m)}\right)=\operatorname{dim}(A(t))=n$.

Proof: The first relation comes from remark 1.8 and the noetherianity hypothesis. The second relation comes from corollary 1.7 and [7, proposition 1.4].

The following result is a consequence of [7, theorem 1.6], lemma 1.6 and corollary 1.10 .

Corollary 1.11 Let $A$ be a noetherian, biequidimensional, universally catenarian $k$-algebra of Krull dimension $n$, such that for any maximal ideal $\mathfrak{m}$ of $A$, the residue field $A / \mathfrak{m}$ is algebraic over $k$. Then every maximal ideal of $A_{(\infty)}$ has height $n$. 


\section{The biggest perfect subfield of a formal function field}

Throughout this section, $k$ will be a perfect field of characteristic $p>0$, $A=k[[\underline{X}]], \mathfrak{p} \subset A$ a prime ideal, $R=A / \mathfrak{p}$ and $K=Q t(R)$.

The aim of this section is to prove that the biggest perfect subfield of $K$, $K^{\sharp}=\bigcap_{e>0} K^{p^{e}}$, is an algebraic extension of the field of constants, $k$. This result is proved in proposition 2.5 and it is one of the ingredients in the proof of corollary 3.8 .

Proposition 2.1 Under the above hypothesis, it follows that $k=R^{\sharp}$.

Proof: Let $\mathfrak{m}$ be the maximal ideal of $R$. It suffices to prove that $R^{\sharp} \subseteq k$. If $f \in R^{\sharp}$, then for every $e>0$ there exists an $f_{e} \in R$ such that $f=f_{e}^{p^{e}}$.

- Suppose at first that $f$ is not a unit, then $f_{e}$ is not a unit for any $e>0$, and $f_{e} \in \mathfrak{m}$ for every $e>0$. Thus, $f \in \mathfrak{m}^{p^{e}}$ for every $e>0$ and by Krull's intersection theorem,

$$
f \in \bigcap_{e \geq 0} \mathfrak{m}^{p^{e}}=\bigcap_{r \geq 0} \mathfrak{m}^{r}=(0)
$$

- If $f$ is unit, then $f=f_{0}+\widetilde{f}$, with $f_{0} \in k \subset R^{\sharp}$ and $\widetilde{f} \in R^{\sharp}$ and $f_{0}$ is unit. By the above case $\tilde{f}=0$, hence $f \in k$.

Proposition 2.2 If $\mathfrak{p}=(0)$, that is $R=k[[\underline{X}]]$ and $K=k((\underline{X}))$, then $k=K^{\sharp}$.

Proof: This is a consequence of prop. 2.1 and the fact that $R$ is a unique factorization domain.

In order to treat the general case, let us look at some general lemmas.

Lemma 2.3 (cf. [3, Chap. 5, §15, ex. 8]) If L is a separable algebraic extension of a field $K$ of characteristic $p>0$, then $L^{\sharp}$ is an algebraic extension of $K^{\sharp}$.

Proof: If $x \in L^{\sharp}$, then $x=y_{e}^{p^{e}}$ with $y_{e} \in L$ for all $e \geq 0$. Since $y_{e}$ is separable over $K, K\left(y_{e}\right)=K\left(y_{e}^{p^{e}}\right)=K(x)$, it follows that $y_{e}=x^{p^{-e}} \in K(x)$ and then $x \in K^{p^{e}}\left(x^{p^{e}}\right)$. Therefore

$$
\left[K^{p^{e}}(x): K^{p^{e}}\right]=\left[K^{p^{e}}\left(x^{p^{e}}\right): K^{p^{e}}\right]=[K(x): K] .
$$

Thus $x$ satisfies the same minimal polynomial over $K^{p^{e}}$ and over $K$ for all $e \geq 0$, and the coefficients of this minimal polynomial must be in $K^{\sharp}$. So $x$ is algebraic over $K^{\sharp}$. 
Lemma 2.4 Every algebraic extension of a perfect field is perfect.

Proof: This is obvious because this is true for the finite algebraic extensions.

Proposition 2.5 Let $k$ be a perfect field of characteristic $p>0, A=$ $k[[\underline{X}]]=k\left[\left[X_{1}, \ldots, X_{n}\right]\right], \mathfrak{p} \subset A$ a prime ideal, $R=A / \mathfrak{p}$ and $K=Q t(R)$. Then $K^{\sharp}$ is an algebraic extension of $k$.

Proof: ${ }^{1}$ Let $r=\operatorname{dim}(A / \mathfrak{p}) \leq n$. By the normalization lemma for power series rings (cf. $[1,24.5$ and 23.7$])^{2}$, there is a new system of formal coordinates $Y_{1}, \ldots, Y_{n}$ of $A$, such that

- $\mathfrak{p} \cap k\left[\left[Y_{1}, \ldots, Y_{r}\right]\right]=\{0\}$,

- $k\left[\left[Y_{1}, \ldots, Y_{r}\right]\right] \hookrightarrow \frac{A}{\mathfrak{p}}=R$ is a finite extension, and

- $k\left(\left(Y_{1}, \ldots, Y_{r}\right)\right) \hookrightarrow K$ is a separable finite extension.

The proposition is then a consequence of proposition 2.2 and lemma $2.3 .^{3}$

Remark 2.6 Actually, under the hypothesis of proposition 2.5, J.M. Giral and the authors have proved that the following stronger properties hold:

(1) If $R$ is integrally closed in $K$, then $K^{\sharp}=k$.

(2) In the general case, $K^{\sharp}$ is a finite extension of $k$.

\section{Noetherianity of $A \otimes_{k} k(t)_{p e r}$}

Throughout this section, $k$ will be a perfect field of characteristic $p>0$, keeping the notations of section 1.

Proposition 3.1 Let $K$ be a field extension of $k$ and suppose that $K^{\sharp}$ is algebraic over $k$. For every prime ideal $\mathcal{P} \in \operatorname{Spec}\left(K_{[\infty]}\right)$ such that $\mathcal{P} \cap k[t]=0$ there exists an $m_{0} \geq 0$ such that $\mathcal{P}_{[m]}$ is the extended ideal of $\mathcal{P}_{\left[m_{0}\right]}$ for all $m \geq m_{0}$.

\footnotetext{
${ }^{1}$ Due to J. M. Giral.

${ }^{2}$ The proof of the normalization lemma for power series rings in [1] uses generic linear changes of coordinates and needs the field $k$ to be infinite. This proof can be adapted for an arbitrary perfect coefficient field (infinite or not) by using non linear changes of the form $Y_{i}=X_{i}+F_{i}\left(X_{i+1}^{p}, \ldots, X_{n}^{p}\right)$, where the $F_{i}$ are polynomials with coefficients in $\mathbb{F}_{p}$.

${ }^{3}$ In particular, if $k$ is algebraically closed, we would have $K^{\sharp}=k$.
} 
Proof: The extension $k[t] \subset K^{\sharp}[t]$ is integral and then $\mathcal{P} \cap K^{\sharp}[t]=0$.

We can suppose $\mathcal{P} \neq(0)$. From Remark 1.8, we have ht $\left(\mathcal{P}_{[i]}\right)=\operatorname{ht}(\mathcal{P})=1$ for every $i \geq 0$. Let $F_{i}\left(t_{i}\right) \in K\left[t_{i}\right]$ be the monic irreducible generator of $\mathcal{P}_{[i]}$. From Corollary 1.2, for each $i \geq 0$ there are two possibilities:

(1) $F_{i} \in K^{p}\left[t_{i}\right]$, then $F_{i+1}\left(t_{i+1}\right)=F_{i}\left(t_{i}\right)^{1 / p}$.

(2) $F_{i} \notin K^{p}\left[t_{i}\right]$, then $\mathcal{P}_{[i+1]}=\left(\mathcal{P}_{[i]}\right)^{e}$ and $F_{i+1}\left(t_{i+1}\right)=F_{i}\left(t_{i}\right)=F_{i}\left(t_{i+1}^{p}\right)$.

Since $\mathcal{P} \cap K^{\sharp}[t]=(0)$,

$$
F_{0}\left(t_{0}\right) \notin\left(\bigcap_{m \geq 0} K^{p^{m}}\right)\left[t_{0}\right]=\bigcap_{m \geq 0} K^{p^{m}}\left[t_{0}\right]
$$

and there exists an $m_{0} \geq 0$ such that $F_{0}\left(t_{0}\right) \in K^{p^{m_{0}}}\left[t_{0}\right]$ and $F_{0}\left(t_{0}\right) \notin$ $K^{p^{m_{0}+1}}\left[t_{0}\right]$.

From (1) we have $F_{i}\left(t_{i}\right)=F_{0}\left(t_{0}\right)^{1 / p^{i}} \in K^{p^{m_{0}-i}}\left[t_{i}\right]$ for $i=0, \ldots, m_{0}-1$ and $F_{m_{0}}\left(t_{m_{0}}\right) \notin K^{p}\left[t_{m_{0}}\right]$. Hence, applying $(2)$ repeatedly we find $F_{j+m_{0}}\left(t_{j+m_{0}}\right)=$ $F_{m_{0}}\left(t_{m_{0}}\right)=F_{m_{0}}\left(t_{j+m_{0}}^{p^{j}}\right)$ and $\mathcal{P}_{\left[j+m_{0}\right]}$ is the extended ideal of $\mathcal{P}_{\left[m_{0}\right]}$ for all $j \geq 1$.

Corollary 3.2 Under the same hypothesis of proposition 3.1, $\mathcal{P}$ is the extended ideal of some $\mathcal{P}_{\left[m_{0}\right]}$.

Proof: This is a consequence of prop. 3.1 and the equality $\mathcal{P}=\bigcup_{m \geq 0} \mathcal{P}_{[m]}$.

Let $B$ be a free algebra over a ring $A$ and $S \subset A$ a multiplicative subset. We denote by $I \mapsto I^{E}, J \mapsto J^{C}$ (resp. $I \mapsto I^{e}, J \mapsto J^{c}$ ) the extensioncontraction process between the rings $A$ or $S^{-1} A$ (resp. $A$ or $B$ ) and the rings $B$ or $S^{-1} B$ (resp. $S^{-1} A$ or $S^{-1} B$ ).

Proposition 3.3 With the notations above, let $\mathcal{P}_{1}$ be a prime ideal in $B$ such that $\mathcal{P}_{1} \cap S=\emptyset$. Let $\mathcal{P}_{0}=\mathcal{P}_{1}^{C}, \mathcal{P}_{1}=\mathcal{P}_{1}^{e}$ and $\mathcal{P}_{0}=\mathcal{P}_{1}^{C}$. If $\mathcal{P}_{1}=\mathcal{P}_{0}^{E}$, then $\mathcal{P}_{1}=\mathcal{P}_{0}^{E}$.

Proof: Let $\left\{e_{i}\right\}$ be a $A$-basis of $B$. Since $\mathcal{P}_{1} \cap S=\emptyset$, it is clear that $\mathcal{P}_{1}^{c}=\mathcal{P}_{1}, \mathcal{P}_{0}^{c}=\mathcal{P}_{0}$ and $\mathcal{P}_{0}=\mathcal{P}_{0}^{e}$. If $\mathcal{P}_{1}=\mathcal{P}_{0}^{E}$, we have

$\mathcal{P}_{1}=\mathcal{P}_{1}^{e c}=\mathcal{P}_{1}^{c}=\left(\mathcal{P}_{0}^{E}\right)^{c}=\left(\mathcal{P}_{0}^{e E}\right)^{c}=\left(\mathcal{P}_{0}^{E e}\right)^{c}=\left(\mathcal{P}_{0}^{E}\right)^{e c}=\sum_{s \in S}\left(\mathcal{P}_{0}^{E}: s\right)_{B} \supset \mathcal{P}_{0}^{E}$.

To prove the other inclusion, take an $s \in S$ and let $f=\sum a_{i} e_{i}$ be an element of $\left(\mathcal{P}_{0}^{E}: s\right)_{B}$ with $a_{i} \in A$. Then, $s f=\sum\left(s a_{i}\right) e_{i} \in \mathcal{P}_{0}^{E}$ and from the equality $\mathcal{P}_{0}^{E}=\left\{\sum b_{i} e_{i} \mid b_{i} \in \mathcal{P}_{0}\right\}$ we deduce that $s a_{i} \in \mathcal{P}_{0}$ and $a_{i} \in\left(\mathcal{P}_{0}^{E}: s\right)_{A}=\mathcal{P}_{0}$. Therefore $f \in \mathcal{P}_{0}^{E}$. 
Proposition 3.4 Let $R$ be an integral k-algebra, $K=Q t(R)$, and suppose that $K^{\sharp}$ is algebraic over $k$. Then any prime ideal $\mathcal{P} \in \operatorname{Spec}\left(R_{[\infty]}\right)$ with $\mathcal{P} \cap k[t]=0$ and $\mathcal{P} \cap R=0$ is the extended ideal of some $\mathcal{P}_{\left[m_{0}\right]}, m_{0} \geq 0$.

Proof: Let us write $T=R-\{0\}$. We have $K=T^{-1} R$ and $K_{[m]}=T^{-1} R_{[m]}$ for all $m \geq 0$ or $m=\infty$. We define $\mathcal{P}=T^{-1} \mathcal{P}$. We easily deduce that $\mathcal{P}_{[m]}=T^{-1} \mathcal{P}_{[m]}$ for all $m \geq 0$.

From proposition 3.1, there exists an $m_{0} \geq 0$ such that $\mathcal{P}_{[m]}$ is the extended ideal of $\mathcal{P}_{\left[m_{0}\right]}$ for every $m \geq m_{0}$. Then, proposition 3.3 tells us that $\mathcal{P}_{[m]}$ is the extended ideal of $\mathcal{P}_{\left[m_{0}\right]}$ for every $m \geq m_{0}$, so $\mathcal{P}=\bigcup \mathcal{P}_{[m]}$ is the extended ideal of $\mathcal{P}_{\left[m_{0}\right]}$.

Proposition 3.5 Let $K$ be a field extension of $k$ and suppose that $K^{\sharp}$ is not algebraic over $k$. Then $K_{(\infty)}$ is not noetherian.

Proof: Let $s \in K^{\sharp}$ be a transcendental element over $k$.

For each $m \geq 0$, let $s_{m}=s^{\frac{1}{p^{m}}} \in K$ and $\alpha_{m}=t_{m}-s_{m}$. Let $P$ be the ideal in $K_{(\infty)}$ generated by the $\alpha_{m}, m \geq 0$. We have $\alpha_{m}=\alpha_{m+1}^{p}$ and $P_{(m)}=K_{(m)} \alpha_{m}$ for all $m \geq 0$.

Suppose that $P$ is finitely generated. Then, there exists an $m_{0} \geq 0$ such that $P=K_{(\infty)} \alpha_{m_{0}}$. By faithful flatness, we deduce that $\alpha_{m_{0}+1} \in$ $K_{\left(m_{0}+1\right)} \alpha_{m_{0}}$. Let us write $\tau=t_{m_{0}+1}, \sigma=s_{m_{0}+1}$. Then, $\alpha_{m_{0}+1}=\tau-\sigma$ and there exist $\psi(\tau) \in K[\tau]=K_{\left[m_{0}+1\right]}, \varphi(\tau) \in k[\tau] \backslash\{0\}$ such that

$$
\varphi(\tau)(\tau-\sigma)=\psi(\tau)(\tau-\sigma)^{p}
$$

Simplifying and making $\tau=\sigma$ we obtain

$$
\varphi(\sigma)=\psi(\sigma)(\sigma-\sigma)^{p-1}=0
$$

contradicting the fact that $s$ is transcendental over $k$. We conclude that $P$ is not finitely generated and $K_{(\infty)}$ is not noetherian.

Theorem 3.6 Let $k$ be a perfect field of characteristic $p>0$ and let $A$ be a $k$-algebra. The following properties are equivalent:

(a) The ring $A$ is noetherian and for any $\mathfrak{p} \in \operatorname{Spec}(A)$, the field $Q t(A / \mathfrak{p})^{\sharp}$ is algebraic over $k$.

(b) The ring $A_{(\infty)}$ is noetherian. 
Proof: Let first prove (a) $\Rightarrow(\mathrm{b})$. By Cohen's theorem (cf. $[6,(3.4)])$, it is enough to prove that any $P \in \operatorname{Spec}\left(A_{(\infty)}\right)-\{(0)\}$ is finitely generated.

From corollaries 1.7 and 1.10, we have

$$
\operatorname{ht}\left(P_{[m]}\right)=\operatorname{ht}\left(P_{(m)}\right)=\operatorname{ht}\left(P_{[\infty]}\right)=\operatorname{ht}(P)=r \leq n .
$$

Consider the prime ideal of $A$ :

$$
\mathfrak{p}:=A \cap P=A \cap P_{[\infty]}=A \cap P_{[m]}=A \cap P_{(m)} .
$$

There are two possibilities (cf. [5, prop. 5.5.3])):

(i) $\operatorname{ht}(\mathfrak{p})=r=\operatorname{ht}\left(P_{[m]}\right)$ and $P_{[m]}=\mathfrak{p}\left[t_{m}\right]$, for every $m \geq 0$.

(ii) $\operatorname{ht}(\mathfrak{p})=r-1=\operatorname{ht}\left(P_{[m]}\right)-1, \mathfrak{p}\left[t_{m}\right] \varsubsetneqq P_{[m]}$ and $A / \mathfrak{p} \varsubsetneqq A\left[t_{m}\right] / P_{[m]}$ is algebraic generated by $t_{m} \bmod P_{[m]}$, for every $m \geq 0$.

In case (i), $P_{[\infty]}$ and $P$ are the extended ideals of $\mathfrak{p}$ and they are finitely generated.

Suppose we are in case (ii). We denote $R=A / \mathfrak{p}, K=Q t(R)$. Then:

$$
R_{[m]}=A_{[m]} / \mathfrak{p}\left[t_{m}\right], \quad R_{[\infty]}=A_{[\infty]} / A_{[\infty]} \mathfrak{p}=A_{[\infty]} / \bigcup_{m \geq 0} \mathfrak{p}\left[t_{m}\right]
$$

Define

$$
\mathcal{P}:=R_{[\infty]} P_{[\infty]}=P_{[\infty]} / \bigcup_{m \geq 0} \mathfrak{p}\left[t_{m}\right] \in \operatorname{Spec}\left(R_{[\infty]}\right) .
$$

We have $\mathcal{P}_{[m]}=\mathcal{P} \cap R_{[m]}=P_{[m]} / \mathfrak{p}\left[t_{m}\right], \mathcal{P} \cap R=\mathcal{P} \cap k[t]=0$ and

$$
\operatorname{ht}\left(\mathcal{P}_{[m]}\right)=\operatorname{ht}\left(P_{[m]} / \mathfrak{p}\left[t_{m}\right]\right)=1, \quad \operatorname{ht}(\mathcal{P})=\operatorname{ht}\left(P_{[\infty]} / \bigcup_{m \geq 0} \mathfrak{p}\left[t_{m}\right]\right)=1
$$

We conclude by applying proposition 3.4: there exists an $m_{0} \geq 0$ such that $\mathcal{P}$ is the extended ideal of $\mathcal{P}_{\left[m_{0}\right]}$. Then, $P_{[\infty]}$ is the extended ideal of $P_{\left[m_{0}\right]}$ and $P=A_{(\infty)} P_{[\infty]}=A_{(\infty)} P_{\left[m_{0}\right]}$ is finitely generated.

Let us prove now $(\mathrm{b}) \Rightarrow(\mathrm{a})$. Since $A_{(\infty)}$ is faithfully flat over $A$, we deduce that $A$ is noetherian.

Let $\mathfrak{p} \in \operatorname{Spec}(A)$ and let $R=A / \mathfrak{p}, K=Q t(R)$. Noetherianity of $A_{(\infty)}$ implies, first, noetherianity of $R_{(\infty)}$, and second, noetherianity of $K_{(\infty)}$. To conclude we apply proposition 3.5 . 
Corollary 3.7 Let $k$ be a perfect field of characteristic $p>0$ and let $A$ be a noetherian $k$-algebra. The following properties are equivalent:

(a) The ring $A_{(\infty)}$ is noetherian.

(b) The ring $\left(A_{\mathfrak{m}}\right)_{(\infty)}$ is noetherian for any maximal ideal $\mathfrak{m} \in \Omega(A)$.

Proof: For $(\mathrm{a}) \Rightarrow(\mathrm{b})$ we use the fact that $\left(A_{\mathfrak{m}}\right)_{(\infty)}=A_{\mathfrak{m}} \otimes_{A} A_{(\infty)}$.

For $(\mathrm{b}) \Rightarrow(\mathrm{a})$, let $\mathfrak{p} \subset A$ be a prime ideal and let $\mathfrak{m}$ be a maximal ideal containing $\mathfrak{p}$. From hypothesis $(\mathrm{b})$, the $\operatorname{ring}\left(A_{\mathfrak{m}}\right)_{(\infty)}$ is noetherian. Then, from theorem 3.6 we deduce that the field $Q t(A / \mathfrak{p})^{\sharp}=Q t\left(A_{\mathfrak{m}} / A_{\mathfrak{m}} \mathfrak{p}\right)^{\sharp}$ is algebraic over $k$. From theorem 3.6 again we obtain (a).

Corollary 3.8 Let $k$ be a perfect field of characteristic $p>0, k^{\prime}$ an algebraic extension of $k$ and $A=k^{\prime}\left[\left[X_{1}, \ldots, X_{n}\right]\right]$. Then, the $\operatorname{ring} A_{(\infty)}=k(t)_{p e r} \otimes_{k} A$ is noetherian.

Proof: It is a consequence of lemma 2.4, proposition 2.5 and theorem 3.6.

Corollary 3.9 Let $k$ be a perfect field of characteristic $p>0$. If $(B, \mathfrak{m})$ is a local noetherian $k$-algebra such that $B / \mathfrak{m}$ is algebraic over $k$, then $B_{(\infty)}=$ $k(t)_{\text {per }} \otimes_{k} B$ is noetherian. In particular, the field $Q t(B / \mathfrak{p})^{\sharp}$ is algebraic over $k$ for every prime ideal $\mathfrak{p} \subset B$.

Proof: Let $k^{\prime}=B / \mathfrak{m}$. By Cohen structure theorem (cf. [5, Chap. 0, th. 19.8.8]), the completion $\widehat{B}$ of $B$ is a quotient of a power-series ring $A$ with coefficients in $k^{\prime}$. Since $\widehat{B}_{(\infty)}$ is also a quotient of $A_{(\infty)}$, we deduce from corollary 3.8 that $\widehat{B}_{(\infty)}$ is noetherian. Since $\widehat{B}$ is faithfully flat over $B$, the $\operatorname{ring} \widehat{B}_{(\infty)}$ is also faithfully flat over $B_{(\infty)}$. So, $B_{(\infty)}$ is noetherian.

The last assertion is a consequence of theorem 3.6.

Corollary 3.10 Let $k$ be a perfect field of characteristic $p>0$. For any noetherian $k$-algebra $A$ such that the residue field $A / \mathfrak{m}$ of every maximal ideal $\mathfrak{m} \in \Omega(A)$ is algebraic over $k$, the ring $A_{(\infty)}$ is noetherian. Furthermore, if $A$ is regular and equicodimensional then $A_{(\infty)}$ is also regular and equicodimensional of the same dimension as $A$.

Proof: The first part is a consequence of corollaries 3.7 and 3.9. For the last part, we use corollary 1.11 , the fact that all $A_{(m)}, m \geq 0$ are regular and of the same (global homological $=$ Krull) dimension $([7$, th. 1.6] and $[2])$. 


\section{References}

[1] Abhyankar, S. S.: Local Analytic Geometry. Academic Press, New YorkLondon, 1964.

[2] BeršteǏn, I.: On the dimension of modules and algebras IX, Direct limits. Nagoya Math. J. 13 (1958), 83-84.

[3] Bourbaki, N.: Éléments de mathématique, Algèbre, Chapitres 4 à 7 . Lecture Notes in Math. 864, Masson, Paris, 1981.

[4] Garling, D. J. H.. A course in Galois theory. Cambridge University Press, Cambridge, 1986.

[5] Grothendieck, A.: Eléments de géométrie algébrique IV: Etude locale des schémas et de morphismes de schémas (première partie). Inst. Hautes Études Sci. Publ. Math. 20, 1964.

[6] Nagata, M.: Local rings. Robert E. Krieger Publishing Co., Huntington, N.Y., 1975.

[7] Narváez-Macarro, L.: A note on the behaviour under ground field extension of quasi-coefficient fields, J. London Math. Soc. 43 (1991), 12-22.

Recibido: 4 de abril de 2002

Magdalena Fernández Lebrón

Departamento de Álgebra

Facultad de Matemáticas, Universidad de Sevilla

P.O. Box 1160, 41080 Sevilla, Spain

lebron@algebra.us.es

Luis Narváez Macarro

Departamento de Álgebra

Facultad de Matemáticas, Universidad de Sevilla

P.O. Box 1160, 41080 Sevilla, Spain

narvaez@algebra.us.es

Dedicated to Prof. José L. Vicente on his sixtieth birthday. Both authors are partially supported by DGESIC, PB97-0723, BFM2001-3207 and FEDER. 